\title{
Studies on Growth, Spectral, Thermal, Mechanical and Optical Properties of 4-Bromoanilinium 4-Methylbenzenesulfonate Crystal: A Third Order Nonlinear Optical Material
}

\author{
Pillukuruchi Kailasam Sivakumar ${ }^{a, b}$, Saravana Kumar ${ }^{b}$, Rangasamy Mohan Kumar $^{a}$, Ramajayam $^{2}$
}

Kanagadurai ${ }^{a}$, Suresh Sagadevan ${ }^{*}$

\author{
${ }^{a}$ Department of Physics, Presidency College, Chennai, 600 005, India \\ ${ }^{b}$ Department of Physics, Misrimal Navajee Munoth Jain Engineering College, Chennai, 600 097, India \\ ${ }^{c}$ Department of Physics, AMET University, Chennai, 603 112, India
}

Received: January 24, 2016; Revised: May 25, 2016; Accepted: June 25, 2016

\begin{abstract}
4-Bromoanilinium 4-methylbenzenesulfonate (4BPTS) single crystal was successfully grown from ethanol by slow evaporation method at room temperature. The structure of grown crystal was confirmed by single crystal X-ray diffraction studies. The presence of functional groups of grown crystal was confirmed by the Fourier transform infrared spectroscopy (FTIR) spectral analysis. UVVisible absorption study was performed on the grown crystal to determine the cut-off wavelength. The thermal stability of the grown crystal was investigated from the Thermogravimetric analysis (TGA)/ Differential thermal analysis (DTA) analysis. The mechanical behaviour of grown crystal was studied by using Vicker's microhardness test. The third order non-linear optical properties of 4BPTS were investigated by Z-scan technique with $\mathrm{He}-\mathrm{Ne}$ laser radiation and the corresponding non-linear refractive index and absorption coefficients were also calculated.
\end{abstract}

Keywords: Single crystal, XRD, Thermal, Micorhardness and Z-scan Technique

\section{Introduction}

In the present day technologically changing society, non-linear optical (NLO) materials are generally useful in the area of optical data storage, lasers, optical signal processing, second harmonic generation etc. Even though varieties of NLO materials exist, their applications are limited due to physical and chemical properties ${ }^{1}$. NLO applications require good quality single crystals which have large NLO coefficient coupled with improved physical parameters. One potentially attractive system, where there is a potential for realizing very large second order non-linear coefficient is based on organic crystals. Organic materials have been receiving much consideration because the NLO responses in this broad class of materials is microscopic in origin, offering a chance to use theoretical modeling coupled with synthetic flexibility to design and produce novel materials ${ }^{2,3}$. Further studies on organic NLO materials have accordingly produced very good materials with highly attractive characteristics. Organic crystals have been widely studied owing to their non-linear optical coefficients being often larger than that of inorganic materials. In addition to large NLO coefficient, an organic NLO crystal should be transparent in the UV region $^{4,5}$. NLO applications require materials with very large macroscopic second order susceptibilities which are usually constituted from molecules with large molecular first hyperpolarizability and oriented in a non-centrosymmetric arrangement ${ }^{6}$. The majority of the commercial materials for second order applications are inorganics, particularly for high power use. Organic materials are apparent as being

* e-mail: sureshsagadevan@gmail.com structurally more diverse and therefore are believed to have more long term promise than inorganics. In the present work, we have investigations on the 4-Bromoanilinium 4-methylbenzenesulfonate (4BPTS) crystals that were grown by slow evaporation technique. The grown crystals were subjected to various characterization methods such as XRD studies, FTIR studies, UV-Visible measurements, TGA/DTA, microhardness and Z-scan technique.

\section{Synthesis and crystal growth}

The analytical grade reagents were used for synthesis of 4BPTS compound. 4- Bromoaniline $\left(\mathrm{C}_{6} \mathrm{H}_{6} \mathrm{BrN}\right)$ and paratouesulfonate acid $\left(\mathrm{C}_{8} \mathrm{~F}_{17} \mathrm{SO}_{3} \mathrm{H}\right)$ in the equimolar ratio (1:1) were dissolved in ethanol at room temperature. The solution was stirred for about 5 hours for reaction to take place completely. The synthesized salt was further purified by repeated recrystallization in ethanol. The purified 4BPTS salt was dissolved in ethanol until saturation occurred at $40^{\circ} \mathrm{C}$. The homogeneously grown solution was prepared by continuous stirring of the solution. The saturated solution thus obtained was further purified and allowed to evaporate at higher temperature. Using repeated recrystallization process the synthesized material was purified. Spontaneous nucleation that occurred led to the production of tiny seed crystals with good transparency. A flawless seed crystal was selected out of these crystals and suspended in the mother solution so as to enable it to evaporate at room temperature. Single crystals with large size were obtained due to the formation of monomers at the seed crystal sites from the mother solution, after the completion of nucleation and 
growth processes. 4BPTS crystal of dimension about $18 \times$ $9 \times 9 \mathrm{~mm}^{3}$ was harvested in a growth period of twenty four days by slow evaporation of the solvent. The photograph of the grown 4BPTS crystal is shown in Figure 1.

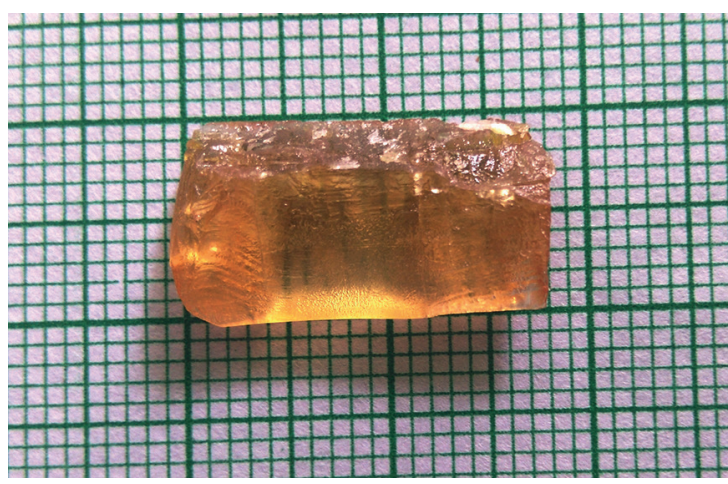

Figure 1: Photograph of as grown 4BPTS single crystal.

\section{Results and Discussion}

\subsection{X-ray diffraction studies}

The unit cell parameters of 4BPTS crystal were found from the single crystal X-ray diffraction analysis using a Bruker kappa APEX II single crystal X -ray diffractometer with $\operatorname{MoK}_{\alpha}(\lambda=0.71073 \AA)$ radiation. The calculated lattice parameters were $\mathrm{a}=5.80 \AA, \mathrm{b}=7.61 \AA, \mathrm{c}=15.95 \AA, \alpha=94.76^{\circ}$, $\beta=96.04^{\circ}, \gamma=92.80^{\circ}$, Volume $(V)=695 \AA^{3}$. The grown crystal belonged to Triclinic crystal system with space group $\mathrm{P}$. The molecular structure and crystal packing of the 4BPTS were shown in Figures 2 and 3.

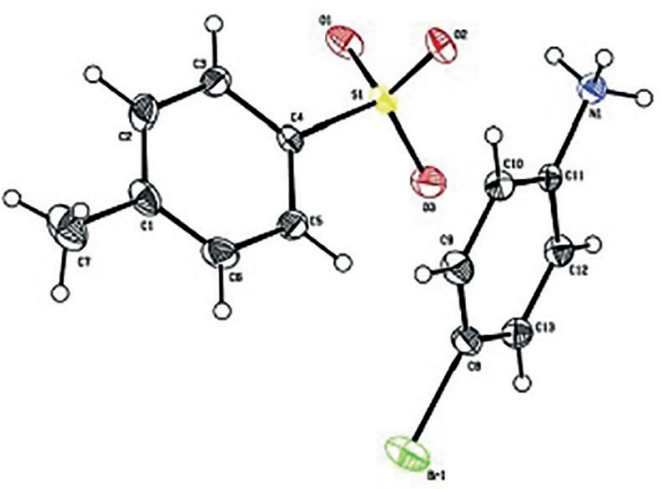

Figure 2: The molecular structure of the 4BPTS.

\subsection{Optical Studies}

The linear optical absorption property was studied using Perkin Elmer Lambda 35 UV-Vis spectrophotometer. The absorption spectrum recorded for the 4BPTS crystal in the region $400 \mathrm{~nm}-900 \mathrm{~nm}$ is shown in Figure 4. UV-VisNIR spectrum gives information about the structure of the

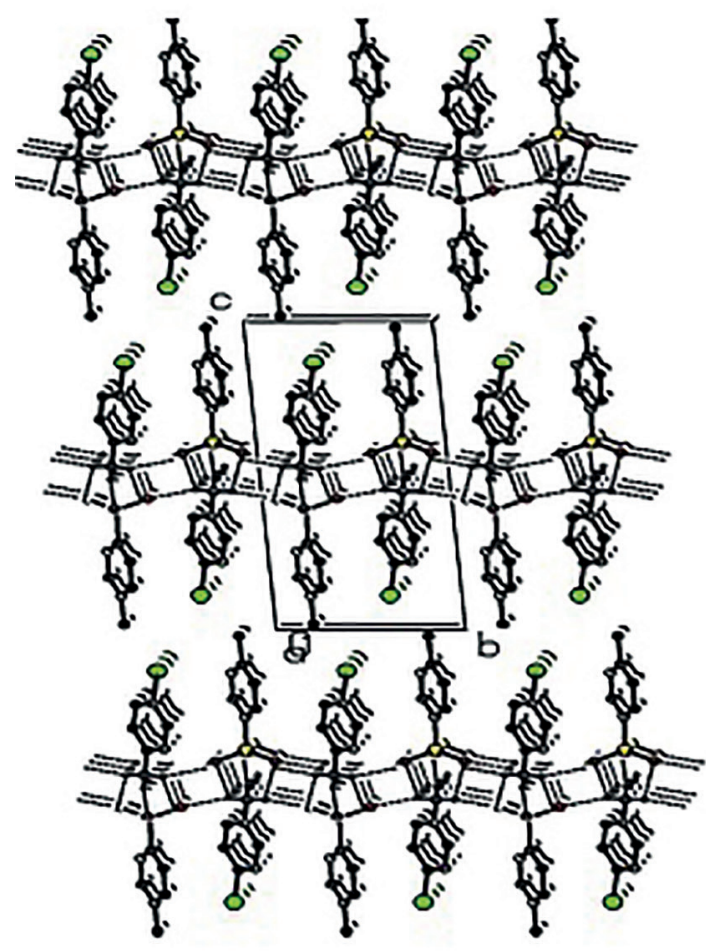

Figure 3: The crystal packing of the 4BPTS.

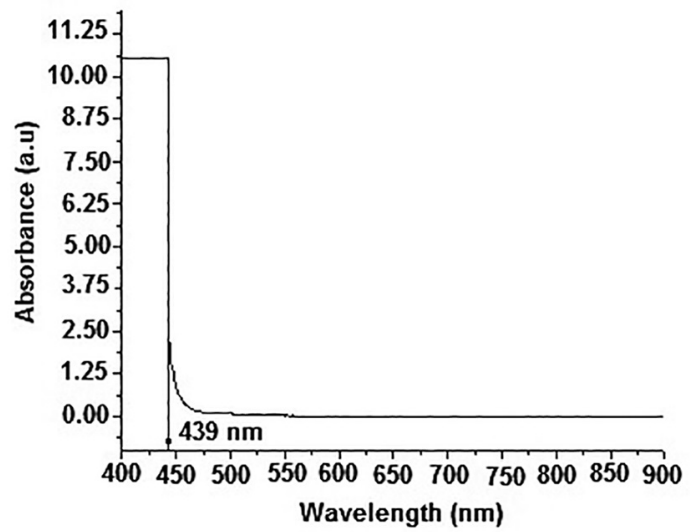

Figure 4: UV-visible absorption spectrum of 4BPTS crystal.

molecule, because the absorption of UV and visible light involves promotion of the electron in the $\sigma$ and $\pi$ orbital from the ground state to higher states. From the spectrum, it is observed that the grown 4BPTS single crystal has the capacity for absorption of light in the region below $439 \mathrm{~nm}$ which is attributed to $\pi-\pi *$ transition due to the presence of conjugated system in the 4BPTS molecule. Optical switching application has required finding a high third order nonlinear optical material, as well as large transmittance or low absorption in near IR region. The internal efficiency of the device also depends upon the absorption coefficient ${ }^{7}$. 


\subsection{FTIR analysis}

It was learnt that the presence of $\mathrm{N}-\mathrm{H}$ in various molecules could be correlated with a constant occurrence of absorption bands whose positions were slightly altered from one compound to another; this was because the atomic group vibrated independently of the other groups in the molecule and had its own frequency. The FTIR spectrum of the grown 4BPTS crystal is shown in Figure 5. The N-H stretching vibrations gave rise to a weak band at $3500-3300 \mathrm{~cm}^{-1}$. In the experimental FT-IR spectrum of 4BPTS a weak band was observed at $3473 \mathrm{~cm}^{-1}$. The $\mathrm{C}-\mathrm{H}$ stretching vibrations of aromatic and hetero aromatic structures occurre ${ }^{8,9}$ in the region $3100-2900 \mathrm{~cm}^{-1}$ for asymmetric stretching modes of vibrations. This permitted the ready identification of the structure. A strong band was observed at $2905 \mathrm{~cm}^{-1}$ in FT-IR that could be assigned to $\mathrm{C}-\mathrm{H}$ stretching respectively. The bands due to $\mathrm{C}-\mathrm{H}$ in-plane bending vibrations were observed in the region $1000-1300 \mathrm{~cm}^{-1}$. For this compound, the $\mathrm{C}-\mathrm{H}$ in-plane bending vibrations were observed at 1127 and 1180 $\mathrm{cm}^{-1}$ in FT-IR spectrum. The C-C stretching modes of the phenyl group were expected in the range from 1650-1200 $\mathrm{cm}^{-1}$. The actual position of these modes was determined not mainly by the nature of the substituents but by the form of substitution around the ring ${ }^{10}$. In the present study, the bands at 1485 and $1508 \mathrm{~cm}^{-1}$ in the FT-IR spectrum were assigned to $\mathrm{C}-\mathrm{C}$ stretching vibration for our 4BTS molecule. Most aromatic Bromo compounds absorb strongly in the region $680-515 \mathrm{~cm}^{-1}$ due to $\mathrm{C}-\mathrm{Br}$ stretching vibrations. The C-Br stretching vibration was assigned to the strong mode 566 $\mathrm{cm}^{-1}$ in the infrared spectra. The identification of $\mathrm{C}-\mathrm{N}$ and $\mathrm{C} \mathrm{N}$ vibrations were a very difficult task, since the mixing of several modes was possible in the region. Silverstein et al., ${ }^{11}$ assigned $\mathrm{C}-\mathrm{N}$ stretching absorption to the region $1266-1382 \mathrm{~cm}^{-1}$ for aromatic amines. In the present work, the bands observed at 1281,1306 , and $1376 \mathrm{~cm}^{-1}$ in the FTIR spectrum were assigned to $\mathrm{C}-\mathrm{N}$ stretching vibrations.

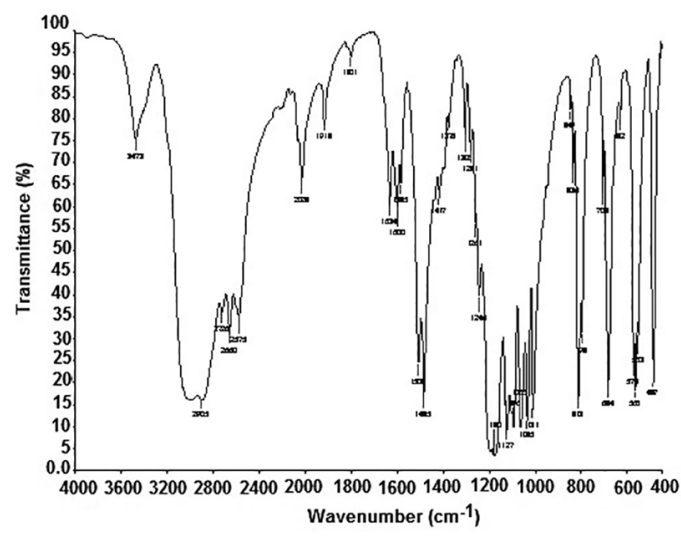

Figure 5: FTIR spectrum of 4BPTS crystal.

\subsection{Thermal Studies}

The thermal behaviour of the crystal was determined by thermo gravimetric analysis and differential thermal analysis using an SDTQ6000V8.2 Built 100 Thermal Analyzer at a heating rate of $10 \mathrm{C} / \mathrm{min}$ in the nitrogen atmosphere in the temperature range of $100-700^{\circ} \mathrm{C}$ as shown in Figure 6 . The TGA curve gives useful information regarding the thermal stability and the composition of the sample under investigation. From the TGA results slight weight loss was observed in the temperature range $100^{\circ} \mathrm{C}-141^{\circ} \mathrm{C}$ and it was due to evaporation of moisture as well as water evaporation. The second drastic weight loss was observed in between $141^{\circ} \mathrm{C}-343^{\circ} \mathrm{C}$ that was due to boiling as well as slight evaporation of $4 \mathrm{BPTS}$. From the DTA results $219.44^{\circ} \mathrm{C}$ the endothermic peak was observed, which was due to the melting of 4BPTS.

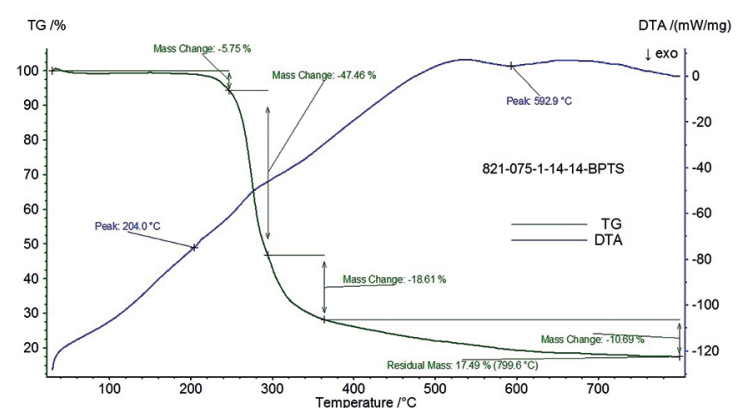

Figure 6: FTIR spectrum of 4BPTS crystal.

\subsection{Vicker's microhardness test}

The structure and the composition of the crystalline solids were inviolably related to the mechanical hardness. Microhardness testing is one of the best methods of understanding the mechanical properties of materials. The microhardness of the grown crystals was measured using a diamond indenter. The well polished crystals were mounted on the platform of the microhardness tester and the load of different magnitudes say, 10, 20 gms were applied over a fixed interval of time. The indentation time was fixed as 5 $\mathrm{s}$. When the load was increased to $100 \mathrm{~g}$ cracks developed on the smooth surface of the crystal due to the release of internal stress generated during indentation. The Vicker's microhardness number was calculated using the relation

$$
H_{V}=\frac{1.8554 P}{d^{2}}\left(\mathrm{~kg} / \mathrm{mm}^{2}\right)
$$

where $\mathrm{P}$ is the applied load in $\mathrm{kg}$ and $\mathrm{d}$ is the average diagonal length in $\mathrm{mm}$. A graph plotted between hardness number $\left(\mathrm{H}_{\mathrm{v}}\right)$ and applied load $(\mathrm{P})$ is shown in Figure 7. The figure shows the variation of load $(\mathrm{P})$ with Vicker's hardness number $\left(\mathrm{H}_{\mathrm{v}}\right)$ for 4BPTS and it was found that Vicker's hardness number increased with the applied load. The initial increase in microhardness (Hv) with increasing load is in agreement with the reverse indentation size effect (RISE).

\subsection{Z - Scan Studies}

The third-order non-linear optical property of 4BPTS crystal was studied by Z-scan technique. The open and closed aperture Z-scan methods were used for the measurement of non-linear absorption coefficient and non-linear optical 


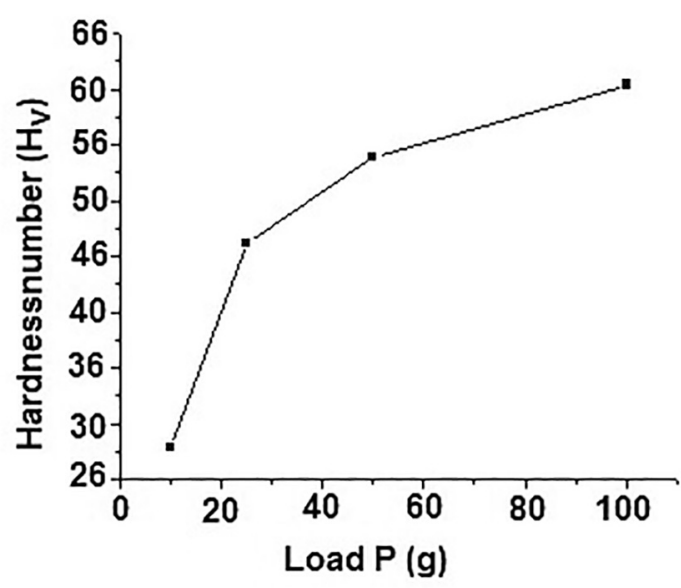

Figure 7: Microhardness number VS Load P.

refraction for optical materials. The magnitude and the sign of the non-linear refractive index $\left(\mathrm{n}_{2}\right)$ and the non-linear absorption coefficient $(\beta)$ of the crystals were calculated from the $\mathrm{Z}$-scan data ${ }^{12}$. By placing the sample in different positions with respect to the focus of the beam, the corresponding normalized transmission to the crystal was measured. Because of the light induced non-linearity, the sample's transmission might increase or decrease, depending on its Z-position with respect to the focal plane. The non-linear absorption coefficient and non-linear optical refraction for the sample were estimated from normalized transmission of corresponding Z-position. The optically polished $1 \mathrm{~mm}$ thick crystal sample was fixed in the travel range of $12 \mathrm{~mm}$. The laser light $632.8 \mathrm{~nm}$ with power of $20 \mathrm{~mW}$ beam from $\mathrm{He}-\mathrm{Ne}$ laser was used. The input energy and the energy transmitted by the sample were measured using a power meter. The peak followed by a valley of normalized transmittance is the signature for non-linearity of the material ${ }^{13,14}$. The Z-scan curves in closed and open aperture modes are illustrated in Figures 8 and 9 respectively. The sample can act as a thin lens of variable focal length, as the sample brought closer to the focus, the irradiance of Gaussian beam increases or decreases depending upon the material absorption and refractive index. From open aperture method, the nonlinear absorption coefficient can be easily calculated from maximum or minimum transmittance curves. It was observed that, the sample transmittance increases while increasing the input intensity of laser beam, which demonstrates the absence of reverse saturation absorption with enhanced strong saturation absorption in 4BPTS crystal. From the closed aperture curve, the prefocal valley to post focal peak configuration clearly suggests that the title crystal has a positive sign of third order nonlinear refractive index and this behaviour attributed to self focusing effect. This may caused by reduced transmittance and large beam divergence through the far field aperture, which is an essential property for optical switching applications.

The difference between the peak and valley transmission $\left(\mathrm{DT}_{\mathrm{p}-\mathrm{v}}\right)$ is written in terms of the on axis phase shift at the focus as,

$$
\Delta T_{p-v}=0.406(1-S)^{0.25}\left|\Delta \varphi_{0}\right|
$$

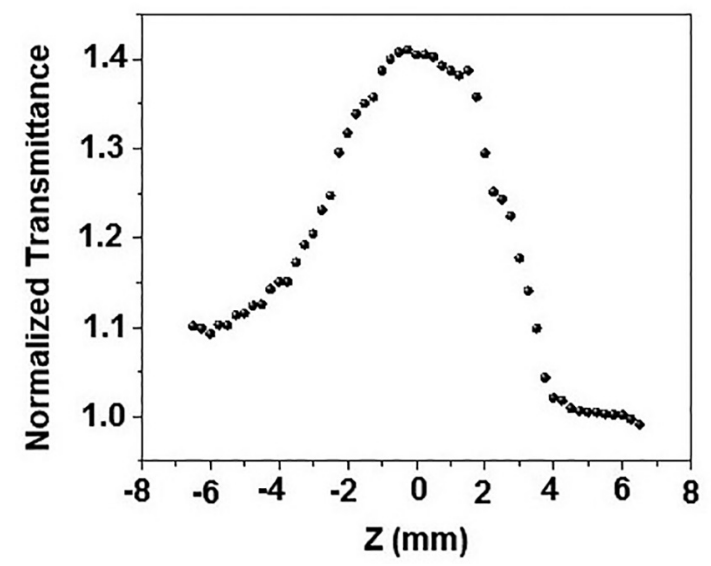

Figure 8: Z-scan open curvature of the 4BPTS.

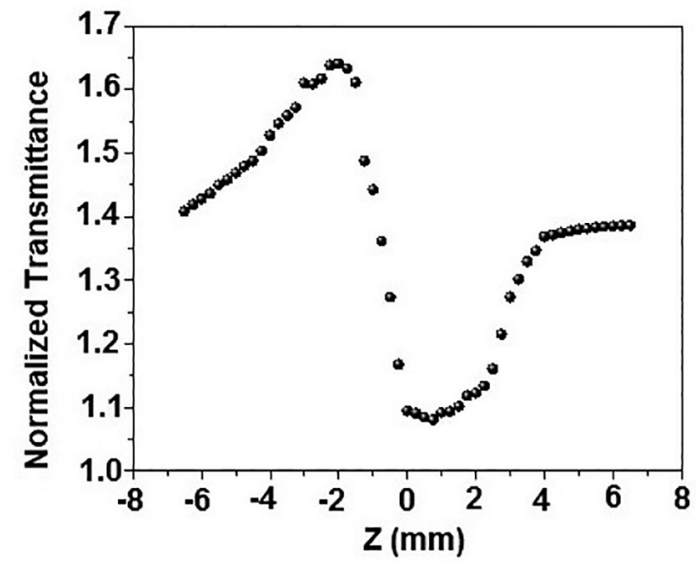

Figure 9: Z-scan close curvature of the 4BPTS.

where $\mathrm{DF}_{0}$ is the on-axis phase shift at the focus, $\mathrm{S}$ is the linear transmittance aperture and it was calculated using the relation,

$$
S=1-\exp \left(\frac{-2 r_{a}^{2}}{\omega_{a}^{2}}\right)
$$

where $r_{a}$ is the radius of aperture and $\mathrm{w}_{\mathrm{a}}$ is the beam radius at the aperture. The non-linear refractive index $\left(\mathrm{n}_{2}\right)$ was calculated using closed aperture Z-scan data and it is given by

$$
n_{2}=\frac{\Delta \varphi_{0}}{k I_{0} L_{\text {eff }}}
$$

where $\mathrm{k}$ is the wavenumber $(\mathrm{k}=2 \pi / \lambda), \mathrm{I}_{0}$ is the intensity of the laser beam at the focus $(Z=0)$ and $L_{\text {eff }}=\{[1-\exp$ $(-\alpha \mathrm{L})] / \alpha\}$ is the effective thickness of the sample, $\alpha$ is the linear absorption and $\mathrm{L}$ is the thickness of the sample. From the open aperture curve, the non-linear absorption coefficient $(\beta)$ was estimated from the following relation

$$
\beta=\frac{2 \sqrt{2 \Delta T}}{I_{0} L_{\text {eff }}}
$$


where DT is the peak value at the measured open aperture. The real and the imaginary parts of the third-order non-linear optical susceptibility $\left(\mathrm{c}^{3}\right)$ were calculated using the following equations

$$
\operatorname{Re} \chi^{(3)}(e s u)=\frac{10^{-4}\left(\varepsilon_{0} C^{2} n_{0}^{2} n_{2}\right)}{\pi}\left(\mathrm{cm}^{2} / w\right)
$$

and

$$
\operatorname{Im} \chi^{(3)}(\text { esu })=\frac{10^{-2}\left(\varepsilon_{0} C^{2} n_{0}^{2} \lambda \beta\right)}{4 \pi^{2}}(\mathrm{~cm} / w)
$$

where $\varepsilon_{0}$ is the vacuum permittivity and $\mathrm{C}$ is the velocity of light in vacuum. The Z-scan measurements revealed that 4BPTS exhibited good third-order non-linear optical properties such as non-linear refractive index and nonlinear absorption coefficient given in Table 1. The values obtained are in good agreement with the earlier reports of piperazinium 4-nitrophenolate monohydrate (PNP): A third order nonlinear optical material ${ }^{15}$.

Table 1: Results of the z-scan technique.

\begin{tabular}{lc}
\hline Non-linear refractive index $\left(\mathrm{n}_{2}\right)$ & $11.12 \times 10^{-12} \mathrm{~m}^{2} / \mathrm{W}$ \\
\hline Non-linear absorption coefficient $(\beta)$ & $1.42 \times 10^{-4} \mathrm{~cm} / \mathrm{W}$ \\
\hline $\begin{array}{l}\text { Third order non-linear optical } \\
\text { susceptibility }\left(\chi^{3}\right)\end{array}$ & $4.162 \times 10^{-8} \mathrm{esu}$ \\
\hline
\end{tabular}

\section{Conclusion}

Third order non-linear optical 4BPTS single crystals were grown by slow evaporation method. The crystal system and cell parameters were evaluated from single crystal X-ray diffraction analysis. The grown crystal belonged to triclinic crystal system with space group $\mathrm{P}$. The necessary functional groups to form the 4BPTS compound were identified using FT-IR spectral studies. Thermal behaviour of the grown crystal was analysed using TG/DTA. From the DTA results $219.44^{\circ} \mathrm{C}$ the endothermic peak was observed, which was due to the melting of 4BPTS. The optical absorption spectrum helped to determine the cut-off wavelength and it was found to be $439 \mathrm{~nm}$. From the microhardness measurements, it was observed that the hardness increased with increase of load until saturation occurred. The NLO test was carried out by Z-scan technique and it affirmed that 4BPTS exhibited the non-linear optical properties.

\section{References}

1. Aggarwal MD, Stephens J, Batra AK, Lal RB. Bulk crystal growth and characterization of semiorganic nonlinear optical materials. Journal of Optoelectronics and Advanced Materials. 2003;5(3):555-562.
2. Mohammed AV, Arulappan JAP, Ganesan ST, Suresh S. Analysis on the Growth and Characterization of a Nonlinear Optical Single Crystal: L-Cystine Dihydrobromide. Materials Research. 2015;18(4):828-832.

3. Suresh S. Growth, optical, mechanical, dielectric and theoretical properties of picolinium maleate NLO single crystal. Optik - International Journal for Light and Electron Optics. 2014;125(12):2826-2829.

4. Suresh S. Synthesis, growth and characterization of L-threonine zinc acetate (LTZA) NLO single crystal. Optik - International Journal for Light and Electron Optics. 2014;125(16):4547-4551.

5. Nithya N, Mahalakshmi R, Suresh S. Investigation on Physical Properties of Semiorganic Nonlinear Optical Glycine Zinc Sulfate Single Crystal. Materials Research. 2015;18(3):581-587.

6. Azeezaa V, Arulappan JAP, Sunitha TG, Koteeswari P, Suresh S. Growth and characterization of nonlinear optical single crystal: 1-cysteine hydrochloride monohydrate (LCB). Acta Physica Polonica A. 2015;123(3):423-430.

7. Suresh S. Optical and electrical studies of non-linear optical crystal: Potassium boro-oxalate. Optik - International Journal for Light and Electron Optics. 2016;127(14):5613-5621.

8. Gunasekaran S, Ponnambalam U, Muthu S, Ponnusamy S. Vibrational and normal coordinates analysis of Pyrazinamide. Asian Journal of Chemistry. 2004;16(3-4):1513-1518.

9. Fulara J, Nowak MJ, Lapinski L, Leś L, Adamowicz L. Theoretical and matrix-isolation experimental study of the infrared spectra of 5-azauracil and 6-azauracil. Spectrochimica Acta Part A: Molecular Spectroscopy. 1991;47(5):595-613.

10. Bellamy LJ. The infrared spectra of complex molecule. $3 \mathrm{ed}$. New York: John Wiley \& Sons; 1975. 433p.

11. Silverstein RM, Bassler GC, Morrill TC. Spectrometric Identification of Organic Compounds. New York: John Wiley \& Sons; 1981.

12. DeSalvo R, Sheik-Bahae M, Said AA, Hagan DJ, Van Stryland EW. Z-scan measurements of the anisotropy of nonlinear refraction and absorption in crystals. Optics Letters. 1993;18(3):194-196.

13. Subashini A, Kumaravel R, Leela S, Evans HS, Sastikumar D, Ramamurthi K. Synthesis, growth and characterization of 4-bromo-4'chloro benzylidene aniline--a third order non linear optical material. Spectrochimica Acta Part A: Molecular Spectroscopy. 2011;78(3):935-941.

14. Sabari Girisun TC, Dhanuskodi S, Mangalaraj D, Phillip J. Synthesis, growth and characterization of bisthiourea zinc bromide for optical limiting applications. Current Applied Physics. 2011;11(3):838-843.

15. Subhashini V, Ponnusamy S, Muthamizhchelvan C, Dhanalakshmi B. Growth and characterization of piperazinium 4-nitrophenolate monohydrate (PNP): A third order nonlinear optical material. Optical Materials. 2013;35(7):1327-1334. 